
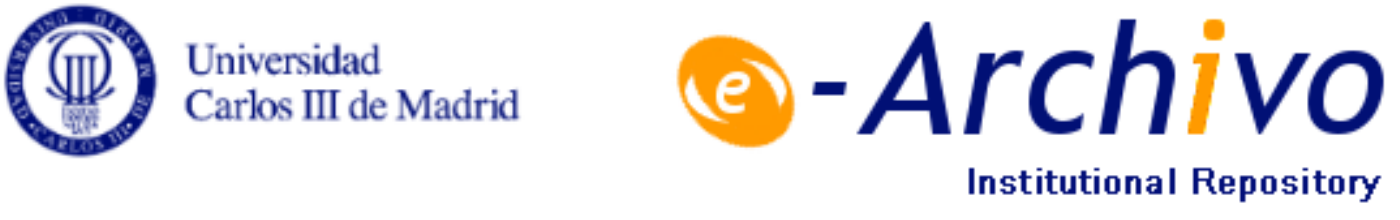

This document is published in:

IEEE Transactions on Consumer Electronics 59 (2013), 1- Febrero PP. 45-53

DOI: 10.1109/TCE.2013.6490240

(C) 2013 IEEE. Personal use of this material is permitted. Permission from IEEE must be obtained for all other uses, in any current or future media, including reprinting/republishing this material for advertising or promotional purposes, creating new collective works, for resale or redistribution to servers or lists, or reuse of any copyrighted component of this work in other works. 


\title{
Energy Efficient Peak Power Reduction in OFDM with Amplitude Predistortion Aided by Orthogonal Pilots
}

\author{
Martha C. Paredes Paredes, Student Member, IEEE, and M. Julia Fernández-Getino García, Member, IEEE
}

\begin{abstract}
The high Peak-to-Average Power Ratio (PAPR) is a main drawback of Orthogonal Frequency Division Multiplexing (OFDM) systems. We propose a twostep technique to reduce the PAPR consisting of a metricbased constellation extension method, such as Simple Amplitude Predistortion (SAP) algorithm, aided by Orthogonal Pilot Sequences (OPS) in a previous step, where we also provide a low-complex implementation of OPS scheme. We show that our proposal, named OP-SAP, outperforms previous approaches in terms of PAPR reduction, due to joining the benefits of Orthogonal Pilots with SAP algorithm. Moreover, it is energy efficient within two aspects: transmitted energy and implementation energy. OP-SAP saves up to $57 \%$ of transmitted energy per predistorted symbol compared to SAP. Regarding implementation energy, PAPR reduction techniques introduce some additional computational complexity, which requires extra cycles in the processor that demand energy consumption. We present an exhaustive analysis on computational power cost that shows the low power consumption of OP-SAP compared to other methods as SeLected Mapping (SLM), what yields a remarkable energy saving in its practical implementation'.
\end{abstract}

Index Terms - OFDM, peak to average power ratio, energy efficiency, computational complexity.

\section{INTRODUCTION}

Orthogonal Frequency Division Multiplexing (OFDM) has been widely adopted in many wireless networks due to its strong immunity to multipath fading, its simple equalizer structure and its high bandwidth efficiency. However, one of the most serious problems is the high Peak-to-Average Power Ratio (PAPR) of the transmitted signal, where the range of PAPR is proportional to the number of subcarriers used in the system. When the signal passes through a nonlinear High Power Amplifier (HPA), high peaks generate in-band distortion, which degrades useful signal, and out-of-band radiation which interferes adjacent channels [1].

This work was supported in part by the Spanish National Projects GRE3N-SYST (TEC2011-29006-C03-03) and COMONSENS (CSD200800010), Fundación Carolina (Spain), and SENESCYT (Ecuador).

M. C. Paredes Paredes, is with the Department of Signal Theory and Communications, Carlos III University of Madrid, Avda. Universidad, 30, 28911-Leganés, Madrid-Spain. (e-mail: mcparedes@tsc.uc3m.es).

M. J. Fernández-Getino García, is with the Department of Signal Theory and Communications, Carlos III University of Madrid, Avda. Universidad, 30, 28911-Leganés, Madrid-Spain. (e-mail: mjulia@tsc.uc3m.es).
The conventional solution to the PAPR problem is to backoff the operating point of the nonlinear HPA, but although simple, this approach usually causes a significant power efficiency penalty. Many methods have been implemented in software and there are several proposals in the literature to suppress the power peaks in OFDM [2]. One simple method is to deliberately clip the OFDM signal before amplification [3]. Clipping can reduce PAPR but this is a nonlinear process that may cause significant in-band distortion, which degrades Bit Error Rate (BER) performance, and out-of-band noise, which reduces spectral efficiency. There are also distortionless techniques such as coding [4], SeLected Mapping (SLM) [5], Partial Transmit Sequences (PTS) [6], [7] and Tone Reservation (TR) [1], [8]. However, these methods either reduce the transmission rate or they may require the transmission of side information to the receiver, which reduces system efficiency. Another class of distortionless PAPR reduction techniques, called nonbijective constellations, tries to alter or introduce new signal constellations to combat large signal peaks without data rate loss such as Active Constellation Extension (ACE) [9] and Tone Injection (TI) [1]. Compared with the previously mentioned techniques, ACE induces no BER degradation and it requires no special processing at reception. However, it introduces an increase in the energy per symbol and it has a high complexity because of an iterative constellation extension process. This complexity burden is significantly reduced with a recent metric-based proposal named Simple Amplitude Predistortion (SAP) [10]. This algorithm predistorts the amplitude of a subset of the input symbols, and, it has been later extended to the phase dimension [11], [12]. Finally, Orthogonal Pilot Sequences (OPS) [13] is another approach that uses two-dimensional Pilot Symbol Assisted Modulation (2D-PSAM), usually employed in coherent OFDM channel estimation, and it is based on inserting known symbols spread throughout the 2D time-frequency grid. These pilot symbols are employed to simultaneously perform distortionless peak power reduction. However, the implementation of OPS [13] requires the use of several Inverse Discrete Fourier Transform (IDFT) operations at the transmitter, yielding a significant complexity load.

All of the previously mentioned schemes require some additional computational complexity beyond the requirements of traditional OFDM, and this additional complexity requires power consumption at the processor. Therefore, it is also of 
high importance to analyze the computational power cost of implementing a PAPR reduction scheme.

In this paper, we address the PAPR reduction in an OFDM system with embedded pilot signaling. In our proposal we use a simple ACE scheme, as it is SAP, aided by OPS technique. By utilizing Orthogonal Pilots combined with SAP, denoted as OP-SAP, we can significantly reduce the transmitted energy per predistorted symbol compared to SAP technique. The amplitude predistortion procedure expands the constellation but we can achieve with OP-SAP a decrease in the scaling factor. The most appealing is that we can obtain at the same time a better performance, in terms of PAPR reduction, than SAP. Therefore, this proposal becomes an energy efficient technique.

Moreover, we present an important improvement in the complexity of OPS compared to its original proposal [13]. We attain decreasing this complexity due to a novel OPS scheme that does not need to carry out additional IDFT-operations at the transmitter, unlike [13].

As pointed out above, the computational complexity introduced by any PAPR reduction scheme is one of the most important aspects in the system, and for this reason, its analysis cannot be ignored in the paper. Indeed, we present an exhaustive calculation of the computational power cost required to implement our proposal and its comparison with other techniques. The saving in computational power cost drives to an extension of battery duration and a reduction in latency time.

The rest of this paper is organized as follows. Section II briefly presents the system model for OFDM and PAPR statistics. In Section III, our proposal OP-SAP is presented, the novel OPS scheme with low-complexity is proposed and transmitted energy is studied. Computational power cost analysis is provided in Section IV, probing the cost saving in the number of required cycles of a Digital Signal Processor (DSP). Performance evaluations and results are discussed in Section V. Finally, conclusions are drawn in Section VI.

\section{THE PAPR OF AN OFDM SIGNAL}

The OFDM signal is the sum of $N$ independent signals modulated onto subchannels of equal bandwidth, which can be efficiently implemented by an IDFT operation. The timedomain signal for the $\ell$ th OFDM symbol is

$$
\mathbf{s}^{\ell}[n]=\frac{1}{\sqrt{N}} \sum_{k=1}^{N-1} S^{\ell}(k) e^{j \frac{2 \pi}{N} k n}, 0 \leq n<N-1
$$

where $k$ and $n$ are the frequency and time indices respectively, and $S^{\ell}(k)$ is the complex symbol transmitted over $k$ th subcarrier, $k=\{0, \cdots, N-1\}$.

The data symbols $S^{\ell}(k)$ are independent, identically distributed (i.i.d.) random variables and due to the central limit theorem, a small percentage of output samples will take very large magnitudes. This results in the well-known PAPR problem of OFDM systems. The PAPR of the time-domain sequence $s\left[{ }^{\ell} n\right]$ associated with the $\ell$ th OFDM symbol is defined as

$$
\chi^{\ell}=\operatorname{PAPR}\left\{s^{\ell}[n]\right\}=\frac{\max \left(\mid s^{\ell}[n]\right)}{E\left\{\mid s^{\ell}[n]^{2}\right\}}
$$

where $\max \left(\left|s^{\ell}[n]\right|\right)$ denotes the maximum instantaneous power and $E\left\{\left|s^{\ell}[n]\right|^{2}\right\}$ denotes the average power of the signal, where $E\{\cdot\}$ denotes expected value.

In the literature, the most common way to evaluate the PAPR is to determine the probability that this PAPR exceeds a certain threshold $\chi_{0}$. This is represented by the Complementary Cumulative Distribution Function (CCDF), which is a random variable [14], given by

$$
\operatorname{CCDF}\left(\chi^{\ell}\right)=\operatorname{Prob}\left(\chi^{\ell}>\chi\right)=1-\left(1-e^{\chi_{0}}\right)^{N}
$$

\section{OP-SAP: AMPlitude Predistortion AIDED BY Orthogonal Pilots}

In order to reduce the PAPR, we propose a two-step technique with SAP algorithm aided by Orthogonal Pilots, making use of a low-complex scheme for OPS. This new architecture is called OP-SAP.

\section{A. Low-complex Orthogonal Pilot Sequences}

In coherent wireless OFDM systems, pilot symbols are usually inserted in the 2D time-frequency grid to estimate the channel. Let us consider that the $\ell$ th OFDM symbol consists of $N$ subcarriers, where a subset $\gamma$ of subcarriers with cardinality $N_{p}=|\gamma|$ will carry pilot symbols and thus, transmitted symbols are

$$
S^{\ell}(k)= \begin{cases}P^{\ell}(k), & k \in \gamma \\ X^{\ell}(k), & k \notin \gamma\end{cases}
$$

where $P^{\ell}(k)$ and $X^{\ell}(k)$ are, respectively, pilot and data symbols at $k$ th subcarrier.

The transmitted discrete-time signal $s^{\ell}[n]=p^{\ell}[n]+x^{\ell}[n]$ can be separated into two parts, as

$$
s^{\ell}[n]=\left\{\begin{array}{l}
p^{\ell}[n]=\frac{1}{\sqrt{N}} \sum_{k \in \gamma} P^{\ell}(k) e^{j \frac{2 \pi}{N} k n} \\
x^{\ell}[n]=\frac{1}{\sqrt{N}} \sum_{k \notin \gamma} X^{\ell}(k) e^{j \frac{2 \pi}{N} k n}
\end{array}\right.
$$

where $p^{\ell}[n]$ and $x^{\ell}[n]$ refer to time-domain pilot and data signals, respectively.

OPS technique [13] proposes the use of a predetermined set of $M$ orthogonal pilot sequences of length $N_{p}\left(M \leq N_{p}\right)$. For the $\ell$ th OFDM symbol, the pilot sequence that provides the lowest PAPR is chosen. This suboptimal scheme reduces 
complexity and avoids any side information compared to the use of optimal values for the pilots. Also, side information is avoided since blind detection is possible at the receiver due to the orthogonality condition [13]. The $N_{p}$ pilot symbols of each OFDM symbol can be collected in a $N$-length sequence denoted as $P^{\ell}$ where, the $k$ th element of this sequence is given by:

$$
\left[P^{\ell}\right]_{k}=\left\{\begin{array}{cc}
P^{\ell}(k), & k \in \gamma \\
0, & k \notin \gamma
\end{array}\right.
$$

As stated before, a set of $M$ pilot sequences are available so the alphabet of $P^{\ell}$ is $\left\{P_{1}, \cdots, P_{M}\right\}$. Each pilot sequence of this finite set $P_{m}, \quad m \in\{1, \cdots, M\}$ contains the frequencydomain pilot symbols at pilot positions while zeros are inserted in the remaining ones. These pilot sequences are orthogonal among them so therefore the ortoghonality condition is fulfilled

$$
\left\langle P_{m}, P_{n}\right\rangle=0 \quad n \neq m \quad m, n=\{1, \cdots, M\}
$$

where $\langle\cdot$,$\rangle denotes the inner product.$

In particular, if the well-known Walsh-Hadamard sequences are employed where $P^{\ell}(k) \in\{1,-1\}$, then $\left\langle P_{m}, P_{n}\right\rangle=N_{p} \delta[m-n], m, n=\{1, \cdots, M\}$ where $\delta[\cdot]$ denotes the Kronecker delta function.

At the transmitter, the original OPS scheme proposed in [13] carries out $M$ IDFT-operations at every $\ell$ th OFDM symbol, as illustrated in Figure 1. However, this frequencydomain implementation yields a significant complexity load.

In our proposal, we avoid $(M-1)$ IDFT-operations at the transmitter by doing time-domain processing. If we realize that the frequency-domain pilot sequences $P_{m}$ can be translated into time-domain as follows

$$
p_{m}[n]=\operatorname{IDFT}\left\{P_{m}\right\}=\frac{1}{\sqrt{N}} \sum_{k \in \gamma}\left[P_{m}\right]_{k} e^{j \frac{2 \pi}{N} k n}
$$

where IDFT $\{\cdot\}$ denotes the IDFT-operation, then the processing at the transmitter can be carried out in timedomain. Firstly, the data signal is translated to time-domain via IDFT operation yielding $x^{\ell}[n]$, that is later combined with the different $p_{m}[n]$, to obtain the transmitted signal, $s_{m}^{\ell}[n]=x^{\ell}[n]+p_{m}[n], m=\{1, \cdots, M\}$.

Also, it must be taken into account that since the pilot sequences are fixed $\forall \ell$, they do not require to be calculated at every OFDM symbol.

The time-domain orthogonal pilot sequences $p_{m}[n]$ can be obtained $\forall m$ at system set-up through their corresponding IDFT calculations over the frequency-domain pilot symbols.
They can be pre-calculated and stored to be later used for every OFDM symbol, unlike [13]. The new low-complex OPS proposal saves calculations, as shown in Figure 2.

For convenience, the OFDM symbol index $(\ell)$ will be omitted in the sequel.

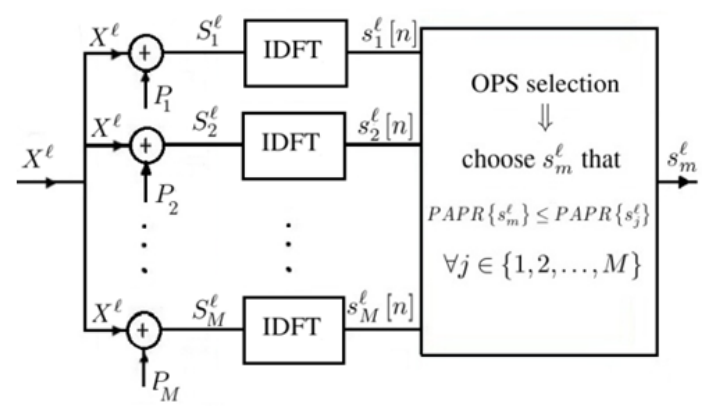

Fig. 1. Original OPS technique [13].

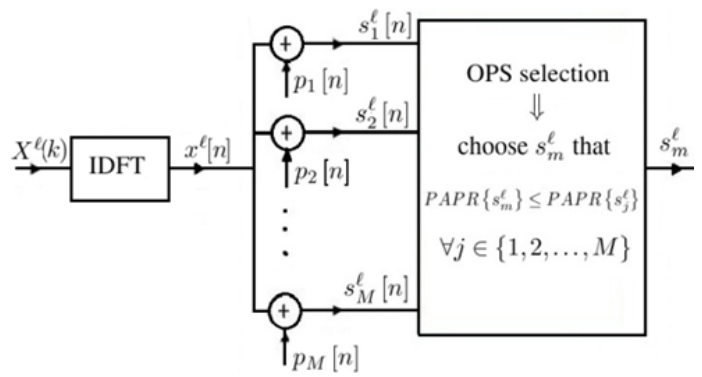

Fig. 2. Low-complex OPS technique.

\section{B. Simple Amplitude Predistortion}

SAP technique predistorts the signal constellation by using a predefined constant scaling factor $\alpha$ without affecting the minimum distance and consequently the system BER. To determine the set of symbols that will be predistorted, the algorithm ([10], [11]) uses a simple metric for each input data symbol that essentially measures how much this symbol contributes to the IDFT output samples with large values. In its general form, the metric for $k$ th input frequency-domain symbol is defined as

$$
\mu_{k}=\sum_{n \in T_{K}} \omega(n) f(n, k)
$$

where, $f(n, k)=-\cos \left(\varphi_{n k}\right)$ is a function which gives an appropriate measure of the phase angle $\left(\varphi_{n k}\right)$ between the output sample $s[n]$ and the contribution of $k$ th symbol $S(k)$, $\omega(n)$ is a weighting function of $s[n]$, defined by $\omega(n)=|s[n]|^{p}$, where $p$ is an appropriately selected parameter and $T_{K}$ is a set of size $K$ whose elements are the indices of the output samples that are larger than a predetermined threshold value $A$. The parameter $A$ should be chosen to include the output samples that are likely to increase the PAPR and becomes close to the target PAPR level after the peak reduction process [11]. 


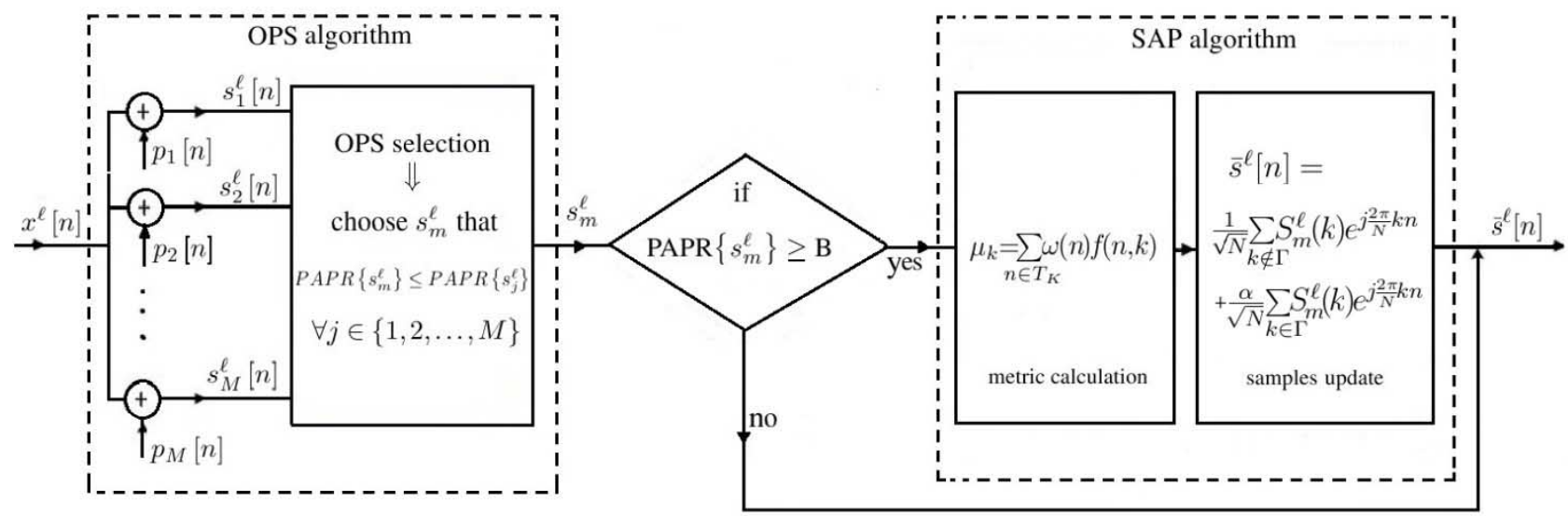

Fig. 3. Block diagram of the transmitter components in OP-SAP technique

Next, the $L$ symbols with greatest positive metrics are determined to be predistorted with their corresponding scaling factor $\alpha>1$. The $\{K, p\}$ parameters are used in the metric calculations, while $\{\alpha, L\}$ are related to the symbol predistortion process.

\section{Proposed Architecture: OP-SAP}

We propose OP-SAP scheme (SAP aided by Orthogonal Pilots, using low-complex OPS) to get a system with a significant improvement in the peak power reduction problem.

The OP-SAP scheme follows the steps that we show in Figure 3. We apply low-complex OPS technique at the first step, this is, the orthogonal pilots are appropriately inserted, to obtain the lowest PAPR from the available set. Then, compute the PAPR and, if this PAPR is larger than a predetermined threshold $B$ (this parameter can be taken as the value for which we obtain the greatest decrease on average PAPR), we apply SAP algorithm. In this case, SAP algorithm is applied over either pilot or data symbols, allowing amplitude predistortion of any complex symbol. The combination of SAP with Orthogonal Pilots is not trivial, since other architectures instead of OP-SAP do not provide any improvement or even a degradation in terms of PAPR reduction, and thus, they were discarded.

The benefits of OP-SAP are that, first, it outperforms previous methods (SAP, SLM) in terms of PAPR reduction. Also, from an efficiency point of view, we can carry out the amplitude predistortion in OP-SAP with less energy per complex symbol than in SAP, if we adequately use the smart pilots to get an energy efficient system. Moreover, we achieve a notable power saving in the additional computational processing due to implementation compared to other schemes (analyzed in Section IV).

The drawbacks of this joint procedure are: (1) it is only intended for coherent systems with pilot symbols, and (2) it implies a slight increase in complexity, compared to SAP alone, since low-complex OPS must be carried out. However, this additional computational burden is negligible since only a search over $M$ sequences is performed.

\section{Transmitted Energy}

The transmitted energy for the $\ell$ th OFDM symbol is $\varepsilon=\varepsilon(0)+\cdots+\varepsilon(N-1)$, where $\varepsilon(k), k=\{0, \cdots N-1\}$, is the energy of the frequency-domain complex symbol, either pilot or data, at $k$ th subcarrier. The choice of the scaling factor in amplitude predistortion techniques obviously has a strong impact on transmitted energy. Let us denote $\Gamma$ as the set of indices of the symbols to be predistorted, this is $|\Gamma|=L$. After amplitude predistortion at $k$ th subcarrier, the energy per symbol becomes $\varepsilon_{p}(k)=\alpha^{2} \varepsilon(k)$. Then, the transmitted energy after predistortion for the $\ell$ th OFDM symbol $\varepsilon_{p}$ is

$$
\varepsilon_{p}=\sum_{k} \varepsilon_{p}(k)=\sum_{k \notin \Gamma} \varepsilon(k)+\alpha^{2} \sum_{k \in \Gamma} \varepsilon(k)
$$

Without amplitude predistortion at any subcarrier $(\alpha=1)$, the transmitted energy $\varepsilon_{p}=\varepsilon$. Considering QPSK modulation with $\varepsilon(k)=1, \forall k$, then, the transmitted energy becomes $\varepsilon_{p}=(N-L)+\alpha^{2} L$. If $\alpha=1$, in the particular case of QPSK, then (10) reduces to

$$
\varepsilon_{p}=\varepsilon=\sum_{k=0}^{N-1} \varepsilon(k)=N
$$

Obviously, we apply OP-SAP algorithm with a lower value of the scaling factor $\alpha$ to attain the same performance compared with the system that uses SAP alone with a greater $\alpha_{0}$ value $\left(\alpha<\alpha_{0}\right)$. In order to examine the saving in terms of transmitted energy between these techniques, we define the ratio of energy $R$, as

$$
R=\frac{\varepsilon_{p}^{O P-S A P}}{\varepsilon_{p}^{S A P}}
$$

where $\varepsilon_{p}^{O P-S A P}$ denotes the transmitted energy per OFDM symbol (after predistortion) when OP-SAP technique is applied, while $\varepsilon_{p}^{S A P}$ refers to the same parameter when SAP technique is considered.

For $k$ th subcarrier, we can also compare the energy per complex symbol between OP-SAP and SAP after amplitude predistortion, as 


$$
R(k)=\frac{\varepsilon_{p}^{O P-S A P}(k)}{\varepsilon_{p}^{S A P}(k)}
$$

\section{Computational Power Cost Analysis}

As it was mentioned above, all PAPR reduction schemes introduce additional computational processing, beyond the requirements of traditional OFDM. This additional processing increases both complexity and power consumption at the processor of an OFDM system. It is reasonable to consider that OP-SAP is not an exception, therefore we present an exhaustive calculation of its computational power cost and we conclude that OP-SAP requires less computational cost than other PAPR reduction schemes. Moreover, a computational cost saving involves less power consumption, let alone the reduction in the latency.

For this analysis we consider a Fixed-Point Digital Signal Processor (DSP) for all computations, and Table I gives a summary of its parameters [15].

Although we have not implemented our technique in a DSP, we have calculated the number of operations required for its practical implementation. Then, based on the parameters of this DSP, we were able to estimate the total number of extra cycles required by the processor, and, therefore, energy consumption, i.e. computational power cost, was determined for this DSP. It must be noted that a reduction of the number of operations optimizes the important design metrics such as area, cost, throughput and power consumption of a device prototype.

TABLE I

RELEVANT DATA FOR FIXED-POINT DSP

\begin{tabular}{lc}
\hline \hline \multicolumn{1}{c}{ Parameter } & Value \\
\hline Current/Processor cycle/second & $0.33 \mathrm{~mA} / \mathrm{MHZ}$ \\
Supply voltage & $1.26 \mathrm{~V}$ \\
Cycles/N-point FFT & $306+5 N / 2 \log _{2}(N / 2)$ \\
Cycles/Multiplication & $1 / 2$ \\
Cycles/Addition & $1 / 4$ \\
Cycles/Complex Multiplication & 3 \\
Cycles $/ X^{*}$-length min-index search & $17+X / 2$ \\
Cycles $/ Y^{*}$-length max-value search & $Y / 2+6$ \\
\hline$X^{*}$ and $Y^{*}$ can take any value
\end{tabular}

\section{A. Total Additional Operations}

Let's consider the total number of operations, due to each PAPR reduction technique, at both transmitter and receiver sides.

\section{a) Transmiter:}

At the transmitter, as seen in Figure 3, in the first stage we have low-complex OPS, which reduces the complexity of OPS [13] avoiding additional IDFT operations and it only needs to carry out $M$ additions, $M N$-length maximum-value searches to find the PAPR of each sequence and a $M$ - length minimum-index search to determine and select the pilot sequence with the lowest PAPR. In the second stage, we propose SAP algorithm, which requires real time operations to calculate the metric given by (9) and then updates the IDFT output samples. The computational cost of the metric calculation step is proportional to $K N$. For QPSK [11], it involves at most $7 \mathrm{KN}$ real multiplications, $3 \mathrm{KN}$ additions and $N$ divisions. Next, update of the time-domain samples has a complexity proportional to $L N$, and for QPSK this step involves $5 L N$ real multiplications and $(2 L+1) N$ additions.

For comparison purposes, the computational power cost of OP-SAP is compared to BSLM (Blind - SLM) [5]. We found that, the BSLM transmitter requires $(D-1)$ additional IDFT operations corresponding to a set of phase sequences, where each sequence is generated on demand [15], and thus $3 \mathrm{~N}$ multiplications are necessary for each sequence. Also, since the data are complex, $N(D-1)$ complex multiplications are necessary. Finally, to select the mapping with minimum PAPR, the transmitter needs $D \quad N$-length maximum-value searches and a $D$ - length minimum-index search.

In Table II we present the additional operations required at the transmitter side by each scheme.

\section{b) Receiver:}

At the receiver, low-complex OPS allows blind detection without requiring any side information to know which pilot sequence was chosen in order to estimate the actual channel [13]. This procedure can be executed with a simple decision rule, based on maximizing the correlation between the equalized signal and the $M$ possible pilot sequences $p_{m}$. On the other hand, SAP does not require any additional calculations. At the BSLM receiver, beyond the DFT necessary in any OFDM system, the receiver must multiply $(D-1)$ inverse phase sequences, then it must perform a minimum-distance maximum-likelihood (ML) decoding to determine which of the constellation points $\left\{C_{q}\right\}_{q=0}^{Q-1}$ (where $Q$ is the number of possible constellation points), that each point, of each mapping correspond to. Finding the square distance between any two points requires 3 additions and 2 multiplications. Therefore, it requires $3 N Q$ additions, $2 N Q$ multiplications and $N Q$-length minimum-index searches for each mapping. In BSLM it is also necessary to identify which phase sequence was used in transmission, so the receiver employs $N D$ additions and a $D$-length minimum-. index search.

In Table II we summarize the total number of operations at the receiver for low-complex OPS, SAP and BSLM.

\section{B. Total Additional Cycles}

If we use the information of Tables I and II, we can calculate the total additional cycles (transmitter and receiver) required by the different techniques: low-complex OPS, SAP, OP-SAP and BSLM.

In general, the total number of cycles is obtained by adding the cycles required by all operations performed at both transmitter and receiver sides. 
TABLE II

ADDITIONAL OPERATIONS FOR EACH TECHNIQUE

\begin{tabular}{|c|c|c|c|c|c|c|}
\hline \multirow{2}{*}{ Operations } & \multicolumn{2}{|c|}{ Low-complex OPS } & \multicolumn{2}{|l|}{ SAP } & \multicolumn{2}{|r|}{ BSLM } \\
\hline & Transmitter & Receiver & Transmitter & Receiver & Transmitter & Receiver \\
\hline IDFT & - & - & - & - & $D-1$ & - \\
\hline Complex multiplications & - & $M N_{p}$ & - & - & $N(D-1)$ & $N(D-1)$ \\
\hline Divisions & - & - & $N$ & - & - & - \\
\hline Additions & $M$ & $M\left(N_{p}+1\right)$ & $N(3 K+2 L+1)$ & - & - & $3 N Q(D-1)+N D$ \\
\hline$D$-length min-index search & - & - & - & - & 1 & 1 \\
\hline$Q$-length min-index search & - & - & - & - & - & $N(D-1)$ \\
\hline$N$-length max-value search & $M$ & - & - & - & $D$ & D \\
\hline$M$-length max-value search & - & 1 & - & - & - & - \\
\hline Overhead cycles & - & - & - & - & $N$ & $N$ \\
\hline
\end{tabular}

Thus, in low-complex OPS, $M N_{p}$ complex multiplications, which need 3 cycles per multiplication, plus $M\left(N_{p}+2\right)$ additions, which need only $1 / 4$ cycle each, plus a $M$-length minimum-index search, which processes $(17+M / 2)$ cycles for each search, plus $M N$-length maximum-value searches, which require $(N / 2+6)$ cycles each, and finally, a $M$ - length maximum-value search, which requires $(M / 2+6)$ cycles. Therefore the total number of cycles in low-complex OPS can be calculated as,

$$
N_{c}^{O P S}=M\left(\frac{7}{4} N_{p}+2\right)+M\left(\frac{1}{2} N+7\right)+2
$$

In order to determine the total cycles of SAP, we should add the $N(7 K+5 L)$ real multiplications and $N$ divisions, which require only $1 / 2$ cycle each; plus $N(3 K+2 L+1)$ additions, which require $1 / 4$ cycle each, as shown in Table II. Next, the total number of cycles for SAP can be written as,

$$
N_{c}^{S A P}=\frac{N}{2}\left(\frac{17}{4} K+3 L+\frac{3}{4}\right)
$$

The total number of cycles in BSLM are: $(D-1)$ IDFT's corresponding to the set of phase sequences, requiring $\left[306+5 N / 2 \log _{2}(N / 2)\right]$ cycles each IDFT, and $6 N(D-1)+2 N Q(D-1)$ real multiplications, needing only $1 / 2$ cycle for each multiplication, plus $2 N(D-1)$ complex multiplications, which require 3 cycles each operation, plus $N Q(D-1)$ additions, which require $1 / 4$ cycle each, plus $(2 D)$ $N$-length maximum-value searches, which require $(N / 2+6)$ cycles each search, plus $2 D$-length minimumindex searches, which process $(17+D / 2)$ cycles each, plus $N(D-1) \quad Q$-length minimum-index searches, which need $(17+Q / 2)$ cycles each, and $2 N$ overhead cycles. Then, the total additional cycles of BSLM are

$$
\begin{aligned}
N_{c}^{B S L M} & =(D-1)\left[\frac{5}{2} N \log _{2}\left(\frac{N}{2}\right)\right]+\frac{N}{4}(41 D-7) \\
& +N(D-1)\left(17+\frac{9}{4} Q\right)+13 D+34
\end{aligned}
$$

Our proposal OP-SAP, as it is a combination of SAP and low-complex OPS, yields a total number of cycles given by

$$
N_{c}^{O P-S A P}=N_{c}^{O P S}+N_{c}^{S A P}
$$

\section{Computational Power Cost}

The computational power cost, also named implementation energy, is measured with the energy consumed by the processor with the extra cycles due to the PAPR technique. Next, we calculate the computational power cost for each scheme, as

$$
E_{T}=N_{c} E_{c}
$$

where $N_{c}$ accounts for the total additional cycles previously computed for each method and $E_{c}$ is the energy consumption per cycle of the processor. Considering the parameters of Table I, $E_{c}$ can be written as:

$$
E_{c}=0.33 \mathrm{~mA} \cdot \mathrm{s} / \mathrm{cycle} \times 1.26 \mathrm{~V}=415.8 \mathrm{pW} \cdot \mathrm{s} / \mathrm{cycle}
$$

This expression particularizes for each method as follows:

$$
\begin{aligned}
E_{T}^{O P S} & =N_{c}^{O P S} \cdot E_{c} \\
E_{T}^{S A P} & =N_{c}^{S A P} \cdot E_{c} \\
E_{T}^{B S L M} & =N_{c}^{B S L M} \cdot E_{c}
\end{aligned}
$$

Finally for our proposal OP-SAP is given by:

$$
E_{T}^{O P-S A P}=N_{c}^{O P-S A P} \cdot E_{c}
$$




\section{Performance Evaluation And Results}

An exhaustive analysis about the performance of OP-SAP, in terms of PAPR reduction (A), transmitted energy (B) and computational power cost $(\mathrm{C})$ is exposed here.

\section{A. PAPR Reduction}

The performance of PAPR reduction was measured by averaging over $10^{5}$ randomly generated complex baseband OFDM symbols with QPSK modulation. We used $N=256$ and 64 subcarriers of which at every $N_{f}$ subcarriers a pilot symbol is inserted. Then, $N_{p}=N / N_{f}$ is the total number of pilot symbols in an OFDM symbol. We consider different values of $M$ for the orthogonal pilot sequences. We employ the well-known Walsh-Hadamard sequences to generate the pilots, where. $P(k) \in\{1,-1\}$. The next step is, if the PAPR is greater than $B=6 \mathrm{~dB}$, computing the metric with $\{K, p=6\}$ parameters set, given at least $A=3.9 \mathrm{~dB}$ power relative to the average signal power.

Our simulation results have been checked with those provided in [5], [10] and [13], what proves that CCDF curves herein are correct and this guarantees the validity of the results.

Figure 4 illustrates the performance of OP-SAP for $N=256$ subcarriers and it is compared with OPS and SAP alone. The improvement is in the order of $1 \mathrm{~dB}$ at a probability of $10^{-2}$

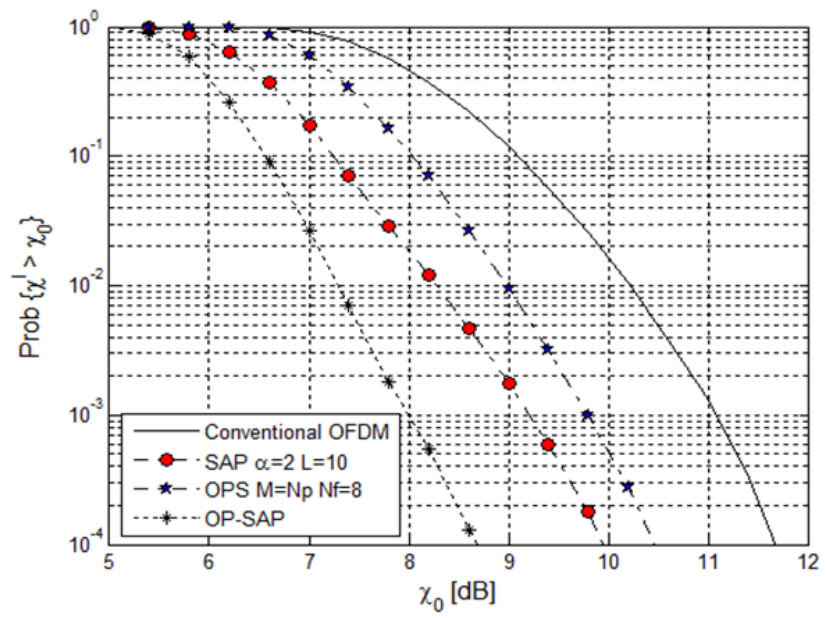

Fig. 4. CCDF of a 256-carrier OFDM system. The solid-line curve corresponds to the conventional OFDM signal without any PAPR reduction technique. The parameters used are: for low-complex OPS $M=N_{p}, N_{p}=32\left(N_{f}=8\right)$, for SAP $\alpha=2$ and $L=10$ symbols and the same parameters for OP-SAP.

Figure 5 shows OP-SAP for $N=256$ while Figures 6 and 7 consider $N=64$. We play with different parameters $\left\{\alpha, L, N_{f}\right\}$. In these figures we observe an improvement in performance compared to BSLM (with $D=4$ ) at a probability of $10^{-2}$, and moreover, we achieve a saving in computational power cost, as we can see in the analysis of Section IV.

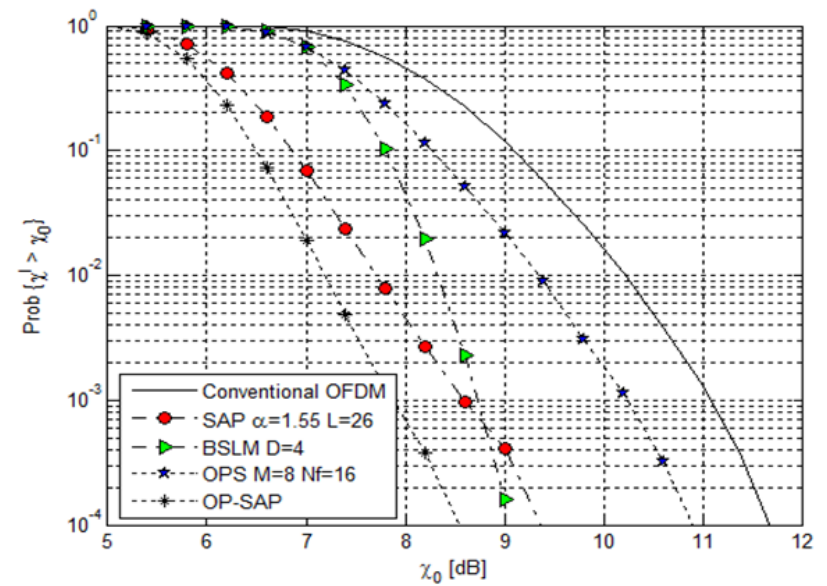

Fig. 5. CCDF of a 256-carrier OFDM system. The solid-line curve corresponds to the conventional OFDM signal without any PAPR reduction technique. Low-complex OPS with parameter $M=8, N_{p}=16$ $\left(N_{f}=16\right)$. SAP with $\alpha=1.55$ and $L=26$ symbols. OP-SAP with the same parameters and BSLM with $D=4$.

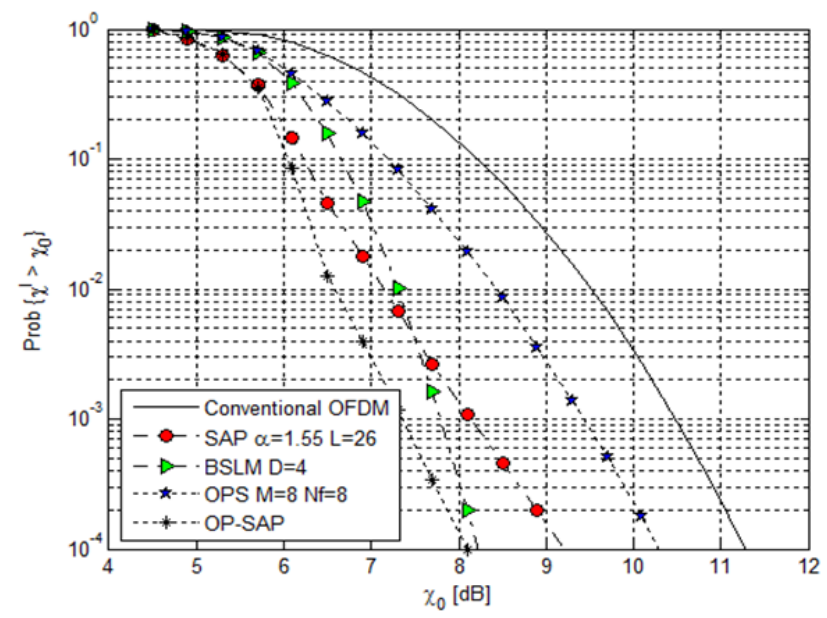

Fig. 6. CCDF of a 64-carrier OFDM system. The solid-line curve corresponds to the conventional OFDM signal without any PAPR reduction technique. Low-complex OPS with $M=8, N_{p}=8\left(N_{f}=8\right)$. SAP with $\alpha=1.55$ and $L=26$ symbols. OP-SAP with the same parameters. BSLM with $\mathrm{D}=4$.

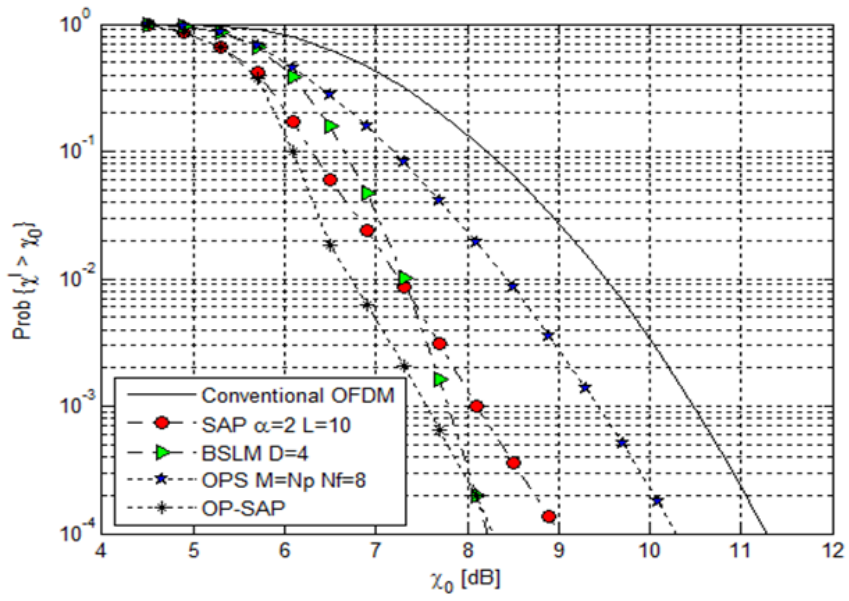

Fig. 7. CCDF of a 64-carrier OFDM system. The solid-line curve corresponds to the conventional OFDM signal without any PAPR reduction technique. Low-complex OPS with $M=N_{p}, N_{p}=8\left(N_{f}=8\right)$, SAP with $\alpha=2$ and $L=10$ symbols. OP-SAP with $N_{p}=8\left(N_{f}=8\right), \alpha=2$ and $L=10$. BSLM with $\mathrm{D}=4$. 


\section{B. Transmitted Energy Efficiency}

Figure 8 demonstrates the benefits of the proposal in terms of transmitted energy for a 256-carrier OFDM system. We obtain similar performance for OP-SAP with $\alpha=1.3$ and SAP with $\alpha=2$, when $L=40$. This means that SAP alone requires a higher value of $\alpha$ (more energy employed for transmission) to guarantee the same performance than OPSAP. The ratio (13) for the kth subcarrier, where $k \in \Gamma$ in this case, is $R(k)=0.4225$, which means that OP-SAP only consumes $42.25 \%$ of the transmitted energy required by SAP.

Then we save up to $57.75 \%$ of the transmitted energy per predistorted symbol.

In Figure 9, we show the energy saving per OFDM symbol that can be obtained with OP-SAP compared to SAP alone, for $N=256$ and $L=40$. In the x-axis we plot the value of $\alpha$ for our proposal, while the $y$-axis provides the difference between the thresholds $\chi_{0}$ (in $\mathrm{dB}$ ) of both schemes at a probability of $10^{-3}$. These results were extracted from Figure 8 . Evidently, if $\Delta=0 \mathrm{~dB}$, it means that both schemes have the same performance. Each curve corresponds to a fixed value of $\alpha$ for SAP alone (given in the legend). In this Figure, we observed that if we apply SAP with $\alpha=2$ we have the same performance than if we use OP-SAP with $\alpha=1.3$. Therefore, for $L=40$ we save up to $24.57 \%$ of energy, calculated as $1-R$, where $R$ is determined using (12).

For $L=26$, we have also found a similar performance between our proposal with $\alpha=1.55$ and SAP with $\alpha=2$. In this case, OP-SAP performance is only slightly better than SAP in terms of PAPR reduction but we still save $13.62 \%$ of energy.

\section{Computational Power Cost}

In Figure 10, we illustrate the computational power cost when implementing the different techniques: low-complex OPS, SAP, BSLM and OP-SAP using (14), (15), (16) and (17) respectively (total additional cycles) for different number of subcarriers $N$. In the calculations we discard the power required by common IDFT/DFT operations necessary in any OFDM system.

Clearly, we observe in (14), (15), (16) and (17) that the values of the parameters that we use in each technique have a strong impact in the total computational power cost. Indeed, in low-complex OPS scheme, the power cost mainly depends on the size of the predetermined set of orthogonal pilot sequences $M$, as shown in Figure 10, but the impact of changing its value is negligible. For example, if we employ a 64-carrier OFDM system with $M=4$ the $E_{T}^{O P S}$ is $0.0861 \mu \mathrm{W} \cdot \mathrm{s}$, while for $M=8$ the $E_{T}^{O P S}$ is $0.1626 \mu \mathrm{W} \cdot \mathrm{s}$.

The computational power cost of SAP mostly depends on the values taken by the parameters $K$ and $L$. The major impact is introduced by $K$ which is used in the metric calculation. Simulation results say us that $K$ gets closer to $N$, when $N$ is greater than 256 subcarriers. In Figure 10, we observe that the impact of changing $L$ is very slight.

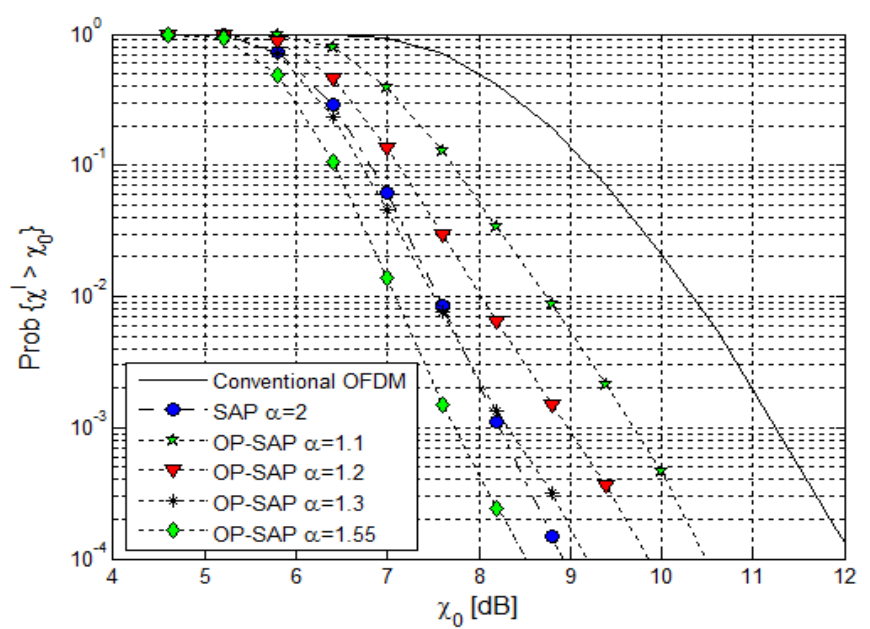

Fig. 8. CCDF of a 256-carrier OFDM system. The solid-line curve corresponds to the conventional OFDM signal without any PAPR reduction technique. SAP and OP-SAP was evaluated for $L=40$ predistorted symbols. OP-SAP is evaluated with different values of $\alpha$ and $M=8, N_{p}=16\left(N_{f}=16\right)$.

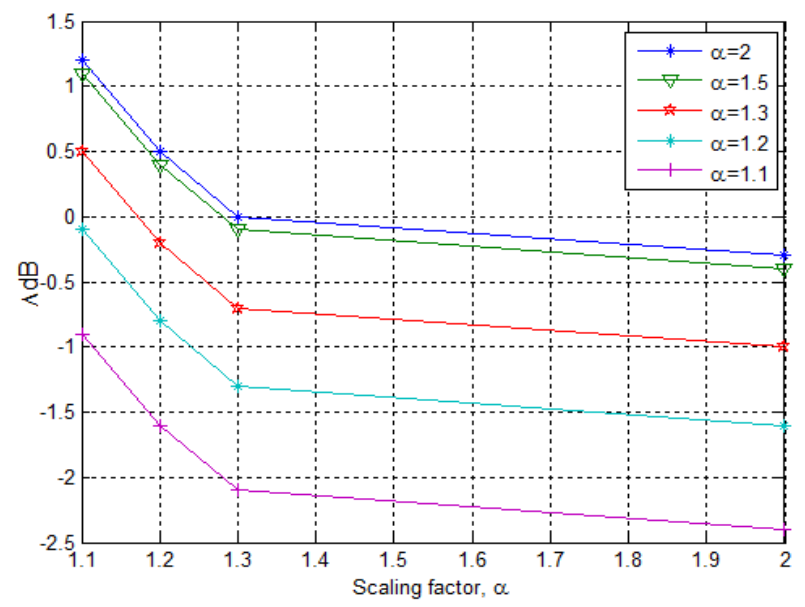

Fig. 9. For a fixed value for SAP alone (legend), performance variation between SAP and OP-SAP given in $\mathrm{dB}$ ( $\mathrm{y}$-axis) for a variable value of $\alpha$ in OP-SAP proposal (x-axis). Negative $\Delta$ values are desired. 256-carrier OFDM system, SAP and OP-SAP with $L=40$.

In SLM or BSLM the additional IDFTs used at the transmitter, which are equal to $D$ phase sequences, produce the major impact in the additional power consumed. As shown in Figure 10, the additional power consumed significantly increases if $D$ grows.

The OP-SAP power cost involves the parameter set of both low-complex OPS and SAP schemes $\{M, K, L\}$ which makes its design more flexible. If we play adequately with these parameters, we attain a good performance in PAPR reduction and also a low power cost.

To evaluate the computational power cost of our proposal, obviously we compare schemes with similar performance in terms of PAPR reduction, as we observe in Figures 6 and 7, that illustrate an OFDM system with $N=64$ subcarriers. In order to calculate the computational power saving of OP-SAP with respect to BSLM, we define the ratio of the computational power costs of both techniques, as 


$$
R_{E_{T}}=\frac{E_{T}^{O P-S A P}}{E_{T}^{B S L M}}
$$

According to the results in Figure 10 and using (24), we obtain that OP-SAP consumes $51.3 \%$ (for parameters of Figure 6) and $58.04 \%$ (for parameters of Figure 7) less implementation energy than BSLM, respectively.

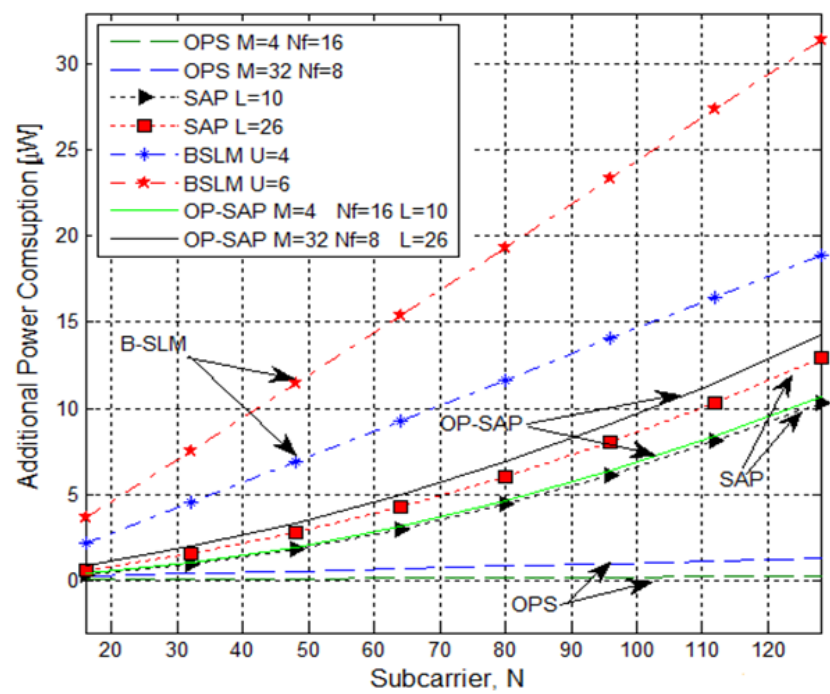

Fig. 10. Computational power cost for each technique.

\section{Conclusions}

We have worked in a new PAPR reduction approach and we explored the benefits of joining SAP scheme with embedded smart pilot signalling, as it is low-complex OPS. We propose a time-domain implementation of OPS with lesscomplexity than the original work. Next, we apply SAP to outperform the performance of previous techniques. With this proposal we can achieve an improvement in PAPR reduction of around $1 \mathrm{~dB}$. Also, we have an energy efficient system due to showing a similar performance than SAP while saving up to $24.57 \%$ in the transmitted energy per OFDM symbol. On the other hand, we obtain a power saving in the computational calculations necessary to carry out any PAPR reduction scheme. While we get a close performance to BSLM, with our proposal we can significantly decrease the computational power cost, obtaining around $58.04 \%$ saving in computational power. Hence, we come to the conclusion that if we embed smart pilots and we combine them with SAP technique we obtain a peak power reduction proposal with relevant savings in transmitted energy and computational power cost, what consequently extends battery life duration.

\section{REFERENCES}

[1] J. Tellado, "Peak to average power reduction for multicarrier modulation," Ph.D. dissertation, Stanford University, Sept. 1999.

[2] T. Jiang and Y. Wu "An overview: Peak-to-average power ratio reduction techniques for OFDM signals, "IEEE Trans. on Broadcasting, vol. 54, no. 2, pp. 257-268, Jun. 2008.

[3] H. G. Ryu, T. P. Hoa, N. T. Hieu, and J. Jin, "BER analysis of clipping process in the forward link of the OFDM-FDMA communication system," IEEE Trans. Consumer Electron., vol. 50, no. 4, pp. 10581064, Nov. 2004.
[4] J. Wen, G. Lee, Ch. Kung, and Ch. Yang, "Coding Schemes Applied to Peak-to-Average Power Ratio (PAPR) Reduction in OFDM Systems," Wireless Communications and Mobile Computing Conference, 2008, IWCMC'08, International, pp. 807-812, 6-8 Aug. 2008.

[5] M. Breiling, S. H. Muller, and J. B. Huber, "SLM peak-power reduction without explicit side information," IEEE Comm. Letters, vol. 5, no. 6, pp. 239-241, Jun. 2001.

[6] S. H. Muller and J. B. Huber, "OFDM with reduced peak-to-averange power ratio by optimal combination of partial transmit sequences," IEE Electronics Letters, vol. 33, no. 5, pp. 368-369, Feb. 1997.

[7] Pooria Varahram and Borhanuddin Mohd Ali, "Partial Transmit Sequence Scheme with New Phase Sequence for PAPR Reduction in OFDM Systems," IEEE Trans. on Consumer Electronics, vol. 57, no. 2, pp. 366-371, May. 2011.

[8] Youngin Park, Sangchae Lim, Dongsoo Har, "Adaptive Phase Rotation of OFDM Signals for PAPR Reduction," IEEE Trans. on Consumer Electronics, vol. 57, no. 4, pp. 1491-1495, Nov. 2011.

[9] B. S. Krongold and D. L. Jones, "PAR Reduction in OFDM via Active Constellation Extension," IEEE Trans. on Broadcasting, vol. 49, no. 3, pp. 258-268, Sept. 2003.

[10] S. Sezginer, and H. Sari, "OFDM Peak Power Reduction with Simple Amplitude Predistortion," IEEE Comm. Letters, vol. 10, no. 12, pp. 6567, Feb. 2006

[11] S. Sezginer, and H. Sari, "Metric-Based Symbol Predistortion Techniques for Peak Power Reduction in OFDM Systems," IEEE Trans. on Wireless Comm., vol. 6, no. 7, pp. 2622-2629, Jul. 2007.

[12] Jwong-Sang Park, Jaen-Kwon Le, and Jin-Up Kim, "Simple Amplitude and Phase Predistortion for PAPR Reduction in OFDM Systems," ETRI Journal, vol. 29, no. 2, pp. 231-233, Apr. 2007.

[13] M. Julia Fernández-Getino García, O. Edfors, and J. M. Páez-Borrallo, "Peak Power Reduction for OFDM Systems With Orthogonal Pilot Sequences," IEEE Trans. on Wireless Comm., vol. 5, no. 1, pp. 47-51, Jan. 2006.

[14] H. Ochiai and H. Imai, "On the Distribution of the Peak-to-Average Power Ratio in OFDM signal," IEEE Trans. on Communications, vol. 49, no.23, pp. 282-289, Feb. 2001.

[15] R. J. Baxley and G. Zhou, "Power savings analysis of peak-to-average power ratio reduction in OFDM," IEEE Trans. on Consumer Electronics, vol. 50, no. 3, pp. 792-798, Aug. 2004.

\section{BIOGRAPHIES}

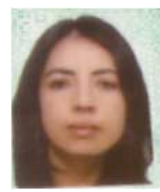

Martha C. Paredes Paredes (S'12) received the Ingeniero en Electrónica y Redes de Información degree from Escuela Politécnica Nacional, Quito, Ecuador in 2008 and the M.Sc. of Multimedia and Communications from Carlos III University of Madrid, Spain in 2010. She is currently pursuing the Ph.D. degree at Carlos III University of Madrid, where she is doing research on signal processing for multicarrier modulation and PAPR reduction in OFDM systems.

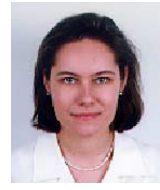

M. Julia Fernández-Getino García (S'99, AM'02, M’03) received the M. Eng. and Ph.D. degrees in telecommunication engineering, both from the Polytechnic University of Madrid, Spain, in 1996 and 2001, respectively. Currently, she is with the Department of Signal Theory and Communications of Carlos III University of Madrid, Spain, as an Associate Professor. From 1996 to 2001, she held a research position at the Department of Signals, Systems and Radiocommunications of Polytechnic University of Madrid. She was on leave during 1998 at Bell Laboratories, Murray Hill, NJ, USA, visited Lund University, Sweden, during two periods in 1999 and 2000, visited Politecnico di Torino, Italy, in 2003 and 2004, and visited Aveiro University, Portugal, in 2009 and 2010. Her research interests include multicarrier communications, coding and signal processing for wireless systems. In 1998 and 2003, she respectively received the best 'Master Thesis' and 'Ph.D. Thesis' awards from the Professional Association of Telecommunication Engineers of Spain, and in 1999 and 2000, she was respectively, awarded the 'Student Paper Award' and 'Certificate of Appreciation' at the IEEE International Conferences PIMRC'99 and VTC'00. In 2004, she was distinguished with the 'Ph.D. Extraordinary Award' from the Polytechnic University of Madrid. In 2012, she has received the 'Excellence Award' to her research from Carlos III University of Madrid. 